\title{
A GENERAL APPROACH TO TOTAL REPAIR COST LIMIT REPLACEMENT POLICIES
}

\author{
FRANK BEICHELT \\ Department of Statistics and Actuarial Science \\ University of the Witwatersrand \\ Johannesburg \\ South Africa
}

\begin{abstract}
A common replacement policy for technical systems consists in replacing a system by a new one after its economic lifetime, i.e. at that moment when its long-run maintenance cost rate is minimal. However, the strict application of the economic lifetime does not take into account the individual deviations of maintenance cost rates of single systems from the average cost development. Hence, Beichelt [2] proposed the total repair cost limit replacement policy: the system is replaced by a new one as soon as its total repair cost reaches or exceeds a given level. He modelled the repair cost development by functions of the Wiener process with drift. Here the same policy is considered under the assumption that the one-dimensional probability distribution of the process describing the repair cost development is given. In the examples analysed, applying the total repair cost limit replacement policy instead of the economic lifetime leads to cost savings of between $4 \%$ and $30 \%$. Finally, it is illustrated how to include the reliability aspect into the policy.
\end{abstract}

\section{INTRODUCTION}

Only a few systems are required to operate without maintenance. They operate in environments such as outer space and high radiation fields, where carrying out maintenance is dangerous, very expensive or simply not possible. Usually, systems are subjected to maintenance, both to preventive and corrective maintenance. In preventive maintenance, systems or their components are exchanged, inspections scheduled, lubricants applied and so on before a failure occurs. In this way, system reliability is preserved or increased by staving off aging effects caused by corrosion, wear, fatigue and other influences. In corrective maintenance, the consequences of system failures are removed by either repairs or replacements. The purpose of preventive maintenance is to reduce the costly and dangerous effects of sudden system 
failures. On the other hand, preventive maintenance gives rise to costs as well. Hence, the problem is to find a cost optimal compromise between preventive and corrective maintenance. The main subject of the mathematical theory of maintenance is to contribute to the solution of this problem, globally and in numerous special situations.

Probably the first cost-based approach towards maintenance optimisation consists in replacing a system by a new one after its economic lifetime, i.e. at that moment, when its long-run total maintenance cost rate (including replacement cost) is minimal (Clapham [4]; Eilon, King, Hutchinson [5]). However, the strict application of the economic lifetime does not take into account individual deviations of maintenance cost rates of systems from the average cost development. Hence, the repair cost limit replacement policy has been proposed: A system is replaced after failure by a new one if the corresponding repair cost exceeds a certain level. Otherwise, a minimal repair is carried out. By definition, a minimal repair does not affect the failure rate of the system, but enables the system to continue its work. (For a survey and discussion, see Beichelt [1]) Thus, even if the total repair cost rate might justify a replacement, the decision to repair or to replace a system depends only on the cost of a single repair. Hence, further cost savings seem to be possible if the whole history of the repair process is taken into account. This leads to the following replacement policy:

Policy 1 The system is replaced as soon as its total repair cost reaches or exceeds a given level c.

In comparison with the repair cost limit replacement policy, policy 1 has two major advantages:

1) Applying policy 1 does not require information on the underlying lifetime distribution of the system.

2) Apart from the pure repair cost, costs due to continuous monitoring, servicing, stock keeping, personnel cost, loan repayment (including interest rates) etc. can be taken into account.

Hence, from the modelling point of view and with respect to its practical implementation, policy 1 is definitely superior to both the economic lifetime approach and the repair cost limit replacement policy. Therefore, in view of its simple structure, and the fact that maintenance cost data are usually available, policy 1 seems to be a suitable basic strategy for planning costoptimal replacement cycles of complex, wear-subjected technical systems such as trucks, 
cranes, caterpillars, belt conveyors etc. and for determining cost-optimal overhaul cycles of whole industrial plants. For obvious reasons, it makes sense to call policy 1 a total repair cost limit replacement policy if the term repair cost includes all maintenance costs apart from replacement costs. Policy 1 was introduced in Beichelt [2]. There repair cost developments are modelled by functions of the Wiener process with drift. This approach allows to generate desired trends of the underlying cost process $\{C(t), t \geq 0\}$ with $C(t)$ being the total repair cost in $[0, t]$. Moreover, the expected first passage times of $\{C(t), t \geq 0\}$, needed for establishing the long-run total maintenance cost rate under policy 1 , can easily be determined. The main result proved in that paper is that applying policy 1 instead of the common economic lifetime approach leads to substantial cost savings. An, at least formal, disadvantage of the Wiener process assumption is that the sample paths of the corresponding cost process $\{C(t), t \geq 0\}$ will decrease in some time intervals, even if functions are chosen in such a way that the trend function of the process $\{C(t), t \geq 0\}$,

$$
M(t)=E(C(t)), \quad t \geq 0,
$$

is fast increasing in $t$. (However, simulation studies indicate that the sample paths of processes $\{C(t), t \geq 0\}$ modelled in this way show a quite realistic behaviour.) This paper pursues the same goal as Beichelt [2], namely to show that, from the cost point of view, policy 1 is superior to the economic lifetime approach. However, it is based on a more adequate theoretical framework. It makes explicit use of the fact that the sample paths of the process $\{C(t), t \geq 0\}$ are non-decreasing. Hence, given the one-dimensional probability distribution of the process $\{C(t), t \geq 0\}$, its first-passage time distribution is given as well. Two models of onedimensional probability distributions are analysed. They indicate the superiority of policy 1 to the economic lifetime approach. (Note that cost comparisons between policy 1 and the repair cost limit policy make little sense since the latter assumes knowledge of the underlying lifetime distribution of the system.) The example distributions (Weibull distribution and a related one) are chosen such that simple explicit formulas for both the repair cost trend function and the expected first passage time exist. Less tractable distributions require the use of numerical methods. For practical applications it is important to point out that, given a set of empirical repair cost data, this paper provides the theoretical basis for determining optimal total repair cost limit replacement policies by simulation. Finally it has to be mentioned that the repair cost processes $\{C(t), t \geq 0\}$ considered in this paper need not be stochastic processes in the strictly mathematical sense. Apart from the problem of its existence, a stochastic process is usually not fully characterized by its one-dimensional probability distribution and the fact that it has non-decreasing sample paths. 


\section{Assumptions}

1) The planning horizon is infinite.

2) Replacements and other maintenance actions take only negligibly small times.

3) The lengths of replacement cycles (times between two neighbouring replacements) are independent, identically distributed random variables with finite expectation.

4) $C(t)$ does not involve replacement costs.

\section{COST CRITERIA}

To determine the long-run total maintenance cost per unit time under policy 1 , we need the first passage time $L(c)$ of the process $\{C(t), t \geq 0\}, C(0)=0$, with respect to a fixed, but arbitrary positive level $c$ :

$$
L(c)=\inf \{t, C(t)>c\}
$$

Since the sample paths of $\{C(t), t \geq 0\}$ are non-decreasing,

$$
P(C(t) \leq c)=P(L(c)>t) \text { for any } c>0 \text { and } t>0
$$

Hence, if $F_{t}(x)=P(C(t) \leq x)$ denotes the probability distribution function of $C(t)$, then the expected value of $L(c)$ is

$$
E(L(c))=\int_{0}^{\infty} F_{t}(c) d t
$$

By the elementary renewal theorem, using assumptions 1 to 4 , the long-run total maintenance cost rate under policy 1 is easily seen to be

$$
K(c)=\frac{a+c}{E(L(c))}
$$

where $a$, the cost of a replacement, is assumed to be constant. Note that $a+c$ is the total maintenance cost within a replacement cycle and $E(L(c))$ is the mean cycle length. The problem consists in finding a maintenance cost limit $c=c^{*}$ which is optimal with respect to $K(c)$. In what follows, policy 1 is compared with policy 2 . Such a comparison makes sense, since policies 1 and 2 have the same cost input and do not require information on the probability distribution of the system lifetime. Moreover, policy 2 is a very common replacement strategy.

Policy 2 The system is replaced by an equivalent new one after $\tau$ time units.

The corresponding long-run total maintenance cost rate is 


$$
K(\tau)=\frac{a+M(\tau)}{\tau}
$$

and the optimal replacement interval $\tau=\tau^{*}$ satisfies equation $K(\tau)=\mathrm{d} M(\tau) / \mathrm{d} \tau$. The time span given by $\tau^{*}$ is called the economic lifetime of the system.

Obviously, it only makes sense to consider probability distributions of $C(t)$ with properties

1) $F_{0}(x)=1$ for all $x \geq 0$

2) $\lim _{t \rightarrow \infty} M(t) / t=\infty$

Property 2 guarantees a sufficiently fast growth of the repair cost trend function $M(t)=E(C(t))$. Otherwise, at least with respect to policy 2, no replacement at all is the cost-optimal behaviour.

\section{DISCUSSION OF SPECIAL CASES}

Example 1 For any positive $t$, let the probability distribution function of $C(t)$ be given by

$$
F_{t}(x)=P(C(t) \leq x)=1-\exp \left\{-\left(\frac{x}{\lambda t^{\alpha}}\right)^{\beta}\right\} ; \lambda>0, \alpha>1, \beta>1 ; x \geq 0
$$

(Weibull distribution). The corresponding probability density is

$$
f_{t}(x)=\frac{\beta}{\lambda^{\beta} t^{\alpha \beta}} x^{\beta-1} \exp \left\{-\left(\frac{x}{\lambda t^{\alpha}}\right)^{\beta}\right\} ; \lambda>0, \alpha>1, \beta>1 ; x \geq 0
$$

Policy 1 According to (1), the probability distribution function of the first passage time $L(c)$ is

$$
P(L(c)>t)=\int_{0}^{c} f_{t}(x) d x
$$

Hence, the expected value of $L(c)$ is given by

$$
E(L(c))=\int_{0}^{\infty} \int_{0}^{c} f_{t}(x) d x d t=\int_{0}^{c} \int_{0}^{\infty} f_{t}(x) d t d x
$$

Changing the order of integration is allowed since the integrand is a continuous function for all $x \geq 0, t>0$ and bounded at $t=0$. Integration yields

$$
E(L(c))=k_{1} c^{1 / \alpha}
$$

with

$$
k_{1}=\left(\frac{1}{\lambda}\right)^{1 / \alpha} \Gamma\left(1-\frac{1}{\alpha \beta}\right)
$$


Hence, the corresponding total maintenance cost rate is

$$
K(c)=\frac{1}{k_{1}} \frac{a+c}{c^{1 / \alpha}}
$$

The optimal limit $c^{*}$ and the corresponding total maintenance cost rate are

$$
c^{*}=\frac{a}{\alpha-1}, \quad K\left(c^{*}\right)=\frac{\alpha}{k_{1}}\left(\frac{a}{\alpha-1}\right)^{(\alpha-1) / \alpha}
$$

Policy 2 The total expected repair cost is

$$
E(C(t))=\int_{0}^{\infty} x f_{t}(x) d x=k_{2} t^{\alpha}
$$

with

$$
k_{2}=\lambda \Gamma\left(\frac{1}{\beta}+1\right)
$$

Hence, when applying the constant replacement interval $\tau$, the total maintenance cost rate becomes

$$
K(\tau)=\frac{a}{\tau}+k_{2} \tau^{\alpha-1}
$$

The corresponding optimal values of $\tau$ and $K(\tau)$ are

$$
\tau^{*}=\left(\frac{a}{k_{2}(\alpha-1)}\right)^{1 / \alpha}, \quad K\left(\tau^{*}\right)=\alpha k_{2}^{1 / \alpha}\left(\frac{a}{\alpha-1}\right)^{(\alpha-1) / \alpha}
$$

Comparison of policies 1 and 2 The inequality $K\left(c^{*}\right)<K(\tau *)$ is easily seen to be equivalent to

$$
1<\Gamma\left(\frac{1}{\beta}+1\right)\left[\Gamma\left(1-\frac{1}{\alpha \beta}\right)\right]^{\alpha}
$$

Note that $[\Gamma(1-1 / \alpha \beta)]^{\alpha}$ is an increasing function in $\alpha, \alpha>1$. For $\alpha=1$, the right-hand side of inequality (8) becomes 


$$
\Gamma\left(\frac{1}{\beta}+1\right) \Gamma\left(1-\frac{1}{\beta}\right)=\frac{1}{\beta} \Gamma\left(\frac{1}{\beta}\right) \Gamma\left(1-\frac{1}{\beta}\right)=\frac{\frac{\pi}{\beta}}{\sin \frac{\pi}{\beta}}
$$

The function $f(x)=x / \sin x$ assumes its infimum in $(0, \pi]$ at $x=0$. Moreover, it is $f(+0)=1$. Hence, inequality (8) holds for all $\alpha, \beta>1$ so that policy 1 is superior to the economic lifetime approach. In particular, if $1.1 \leq \alpha \leq 5$ and $\beta=2$, then average cost savings of between $31 \%$ and $4.2 \%$ are achieved by applying the optimal cost limit $c^{*}$ instead of the economic lifetime $\tau^{*}$.

Example 2 For any nonnegative $t$, let the probability distribution function of $C(t)$ be given by

$$
\begin{aligned}
& F_{t}(x)=P(C(t) \leq x)=\exp \left\{-\left(\frac{\lambda t^{\alpha}}{x}\right)^{\beta}\right\} ; \lambda>0, \alpha>1, \beta>1 ; x>0 \\
& f_{t}(x)=\beta \lambda^{\beta} t^{\alpha \beta} x^{-(\beta+1)} \exp \left\{-\left(\frac{\lambda t^{\alpha}}{x}\right)^{\beta}\right\} ; \lambda>0, \alpha>1, \beta>1 ; x>0
\end{aligned}
$$

Policy 1 Using (3), the expected value of $L(c)$ is seen to be

$$
E(L(c))=k_{1} c^{1 / \alpha}
$$

where

$$
k_{1}=\left(\frac{1}{\lambda}\right)^{1 / \alpha} \Gamma\left(\frac{1}{\alpha \beta}+1\right)
$$

Hence, the total maintenance cost rate again has structure (4) so that the optimal values of $c$ and $K(c)$ are given by (5) with $k_{1}$ given by (9).

Policy 2 The expected maintenance cost is

$$
E(C(t))=k_{2} t^{\alpha}
$$

where

$$
k_{2}=\lambda \Gamma\left(1-\frac{1}{\beta}\right)
$$

With $k_{2}$ given by (10), the optimal values of $\tau$ and $K(\tau)$ are again given by (7).

Comparison of policies 1 and 2 The inequality $K\left(c^{*}\right)<K(\tau *)$ is equivalent to

$$
1<\Gamma\left(1+\frac{1}{\beta}\right)\left[\Gamma\left(1-\frac{1}{\alpha \beta}\right)\right]^{\alpha}
$$


Analogously to example 1 , it can be shown that this inequality holds for all $\alpha \geq 1, \beta \geq 1$, so that policy 1 is again superior to policy 2 . In particular, if $1 \leq \alpha \leq 5$ and $\beta=2$, then average cost savings between $33 \%$ and $6.2 \%$ are achieved when applying policy 1 instead of policy 2 .

\section{COMBINED AGE-TOTAL REPAIR COST LIMIT REPLACEMENT POLICY}

Scheduling replacements on the basis of a total repair cost limit does not take into account, at least not explicitly, reliability requirements imposed on the system. There are several possibilities for including the reliability aspect into the model. A simple way consists in limiting the length of a replacement cycle by a constant $\tau$. By suitably choosing $\tau$ severe breakdowns of the system can be avoided with a given probability. This leads to the following replacement policy.

Policy 3 The system is replaced as soon as the total repair cost reaches level cor after $\tau$ time units, whichever occurs first.

Note that, notwithstanding a formal analogy from the modelling point of view, this policy strongly deviates from the common life-time based "age replacement policy".

Following policy 3 , the probability distribution function of the cycle length,

$$
Y=\min (L(c), \tau)
$$

is, with the notation introduced in the previous sections,

$$
P(Y \leq t)= \begin{cases}1-F_{t}(c), & t \leq \tau \\ 1, & t>\tau\end{cases}
$$

so that

$$
E(Y)=\int_{0}^{\tau} F_{t}(c) d t
$$

In view of assumptions 1 to 3 of section 1, the long-run maintenance cost rate has structure

$$
K(c, \tau)=\frac{[a+E(C(\tau) \mid L(c) \geq \tau)] P(L(c) \geq c)+[a+c] P(L(c)<\tau)}{E(Y)}
$$

Letting $\bar{F}_{\tau}(x)=1-F_{\tau}(x)$,

$$
E(C(\tau) \mid L(c) \geq \tau)=E(C(\tau) \mid C(\tau)<\tau)=\frac{1}{F_{\tau}(c)} \int_{0}^{c} \bar{F}_{\tau}(x) d x
$$

Hence, the long-run total maintenance cost rate becomes 


$$
K(c, \tau)=\frac{a+c \bar{F}_{\tau}(c)+\int_{0}^{c} \overline{F_{\tau}}(x) d x}{\int_{0}^{\tau} F_{t}(c) d t}
$$

Since $\tau$ arises from meeting specific reliability requirements, it is a fixed parameter. Generally, numerical methods have to be applied to minimize $K(c, \tau)$ with respect to $c$

\section{CONCLUSIONS}

The stochastic models for the repair cost development analysed in in this paper as well as some more models not discussed here (Beichelt [3]), give strong arguments in favour of scheduling replacements on the basis of total repair cost limits instead of the economic lifetime. However, much more theoretical and experimental work, in particular Monte-Carlosimulation, needs to be done to get a deeper insight into the relationship between policies 1 and 2. By including the age replacement concept into the total repair cost limit replacement policy, reliability requirements can be taken into account.

\section{REFERENCES}

[1] Beichelt, F. (1993). "A Unifying Treatment of Replacement Policies with Minimal Repair”, Naval Research Logistics, Vol. 40, No. 1, pp. 51-67.

[2] Beichelt, F. (1997). "Total Repair Cost Limit Replacement Policies", ORiON, Vol. 13, No $1 / 2$, pp 37-44.

[3] Beichelt, F. (2000). "A Replacement Policy Based on Cost Restrictions", Lecture at the $2^{\text {nd }}$ Int. Conf. On Mathematical Methods in Reliability Theory, Bordeaux, $3^{\text {rd }}-7^{\text {th }}$ July, 2000.

[4] Clapham, J. C. R. (1957), "Economic life of equipment", Operations Research Quarterly, Vol. 8, No. 2, pp. 181-190.

[5] Eilon, S.; King, J. R.; Hutchinson, D. E. (1966), “A study in equipment replacement”, Operations Research Quarterly, Vol. 17, No. 1, pp. 59-71.

\section{ACKNOWLEDGEMENT}

The author is grateful to a referee and the Editor for many valuable comments on the original draft of the paper. 